\title{
Lung adenocarcinoma patients have higher risk of SARS-CoV-2 infection
}

\author{
Long Chen ${ }^{1}$, Yue Liu ${ }^{1}$, Jiamin $\mathrm{Wu}^{3}$, Chao Deng ${ }^{2}$, Jianjun Tan ${ }^{2}$, Huawen Liu ${ }^{2}$ Li Zhong ${ }^{1}$ \\ ${ }^{1}$ Bioengineering Institute of Chongqing University, Chongqing, China \\ ${ }^{2}$ Three Gorges Central Hospital, Wanzhou, Chongqing, China \\ ${ }^{3}$ Chongqing Mix Biotechnology Co., Ltd, Chongqing, China
}

Correspondence to: Li Zhong, Huawen Liu; email: jlzhong@cqu.edu.cn, 20141902006@cqu.edu.cn

Keywords: lung adenocarcinoma, SARS-CoV-2, ACE2, miR-125b-5p, IL6, COVID-19

Received: June 11, $2020 \quad$ Accepted: July 30, $2020 \quad$ Published: January 10, 2021

Copyright: (c) 2021 Chen et al. This is an open access article distributed under the terms of the Creative Commons Attribution License (CC BY 3.0), which permits unrestricted use, distribution, and reproduction in any medium, provided the original author and source are credited.

\section{ABSTRACT}

Both lung adenocarcinoma and coronavirus disease 2019 would cause pulmonary inflammation. Angiotensinconverting enzyme 2, the functional receptor of SARS-CoV-2, also plays a key role in lung adenocarcinoma. To study the risk of SARS-CoV-2 infection in lung adenocarcinoma patients, mRNA and microRNA profiles were obtained from The Cancer Genome Atlas and Gene Expression Omnibus followed by bioinformatics analysis. A network which regards angiotensin-converting enzyme 2 as the center was structured. In addition, via immunological analysis to explore the essential mechanism of SARS-CoV-2 susceptibility in lung adenocarcinoma. Compared with normal tissue, angiotensin-converting enzyme $\mathbf{2}$ was increased in lung adenocarcinoma patients. Furthermore, a total of 7 correlated differently expressed mRNAs (ACE2, CXCL9, MMP12, IL6, AZU1, FCN3, HYAL1 and IRAK3) and 5 correlated differently expressed microRNAs (miR-125b-5p, miR-9-5p, miR-130b-5p, miR-381-3p and miR-421) were screened. Interestingly, the most frequent toll-like receptor signaling pathway was enriched by mRNA (interlukin 6) and miRNA (miR-125b-5p) sets simultaneously. In conclusion, it was assumed that miR125b-5p-ACE2-IL6 axis could alter the risk of SARS-CoV-2 infection in lung adenocarcinoma patients.

\section{INTRODUCTION}

In December 2019, there was an outbreak of coronavirus pneumonia (COVID-19) caused by SARS-CoV-2 in Wuhan, Hubei province in China. SARS-CoV-2 could encode spike (S) protein like SARS-CoV. In addition, a same cell entry receptor and manner were used to mediate infection. Unlike other type I fusion proteins, the $\mathrm{S}$ protein of coronaviruses is not cleaved in the virusproducing cell $[1,2]$. However, in processed coronaviruses, two domains (S1 and S2) with different functions can be defined [3]. The S1 domain induces receptor association, whereas the S2 domain likely undergoes structural rearrangements that mediate membrane fusion. A discrete receptor-binding domain (RBD) of S protein has been defined at residues 318-510 of the S1 domain. This RBD could bind receptor with higher affinity than full S1 domain $[4,5]$.
Angiotensin-converting enzyme 2 (ACE2), a terminal carboxypeptidase, catalyzes the conversion of angiotensin II (Ang II) to angiotensin 1-7 (Ang 1-7). Ang II, the major effector molecule of the conventional reninangiotensin system, is implicated in the pathogenesis of cardiovascular disorders like hypertension, atherosclerosis, and myocardial infarction. Whereas ACE2 and its product Ang 1-7 are thought to prevent the detrimental effects of angiotensin II [6]. In respiratory organs, ACE2 is the receptor for SARS-CoV [7, 8]. As a type I transmembrane protein, ACE2 is comprised of a short cytoplasmic domain, a transmembrane domain, and a large ectodomain [9]. The ACE2 ectodomain, includes the first $\alpha$-helix and $\mathrm{N}$-terminus proximal residues of $\beta$ sheet, could bind the SARS-CoV S glycoprotein with high affinity [10]. Furthermore, once SARS-CoV binds to ACE2, the abundance on the cell surface, the mRNA expression and enzymatic activity of ACE2 are 
significantly reduced. ACE2 has also been shown to attenuate inflammation and acute lung injury caused by SARS-CoV infection mainly through inhibition of angiotensin II/NF- $\mathrm{KB}$ signaling [11-15]. The ACE2 dysfunction is implicated in SARS pathogenesis. ACE2 is predominantly localized on the apical surface of welldifferentiated airway epithelia, especially ciliated cells, and also been identified with the pathology of various inflammatory lung diseases like lung cancer [16-18]. Furthermore, the expression of ACE2 might be influenced by many factors like organ, gender, age, development, lifestyle (cigarette smoking, diet) and some diseases (chronic obstructive pulmonary disease, hypertension, coronary heart disease, chronic kidney disease and diabetes) [19]. Abundant clinical data has verified that the ACE2 level would alter infection risk of SARS-cov-2. For example, COVID-19 patients with comorbidities were more likely to progress to critically ill patients compared with those without comorbidities [20].

Lung adenocarcinoma (LUAD), the most common primary lung cancer which falls under the umbrella of non-small cell lung cancer (NSCLC), has a strong association with smoking. LUAD usually evolves from the mucosal glands and represents about $40 \%$ of lung cancers. LUAD usually occurs in the lung periphery, and in many cases, may be found in scars or areas of chronic inflammation [21, 22]. The tumor microenvironment of
LUAD may also regulate ACE2 expression level which alters the risk of SARS-CoV-2 infection. Furthermore, there are lots of factors that could cause both ACE2 level alteration and fluctuation of immune-regulation. Thus, through bioinformatics analysis and network structuring, the potential mechanism of COVID-19 in LUAD patient might be explored.

\section{RESULTS}

\section{The expression level of ACE2 in LUAD}

Ang 1-7, the product of ACE2, was confirmed to inhibit the proliferation of human lung cancer cells through interaction with the Mas receptor (MasR) [23]. Ang1-7 not only reduces the size of human lung tumor xenografts in vivo but also markedly decreases their vessel density [24-26]. Thus, the expression level of ACE2 in LUAD would influence the cancer progression. To compare the ACE2 level between LUAD and normal tissues, sequencing data of a total of 830 samples (347 normal tissues and 483 LUAD tissues) were obtained from the TCGA database (Figure 1). In LUAD patients, a higher expression level of ACE2 was detected while there was no significant difference among various stages of LUAD. Due to the function of combining with $S$ glycoprotein, higher ACE2 level might lead to increased risk of SARS-CoV-2 infection.

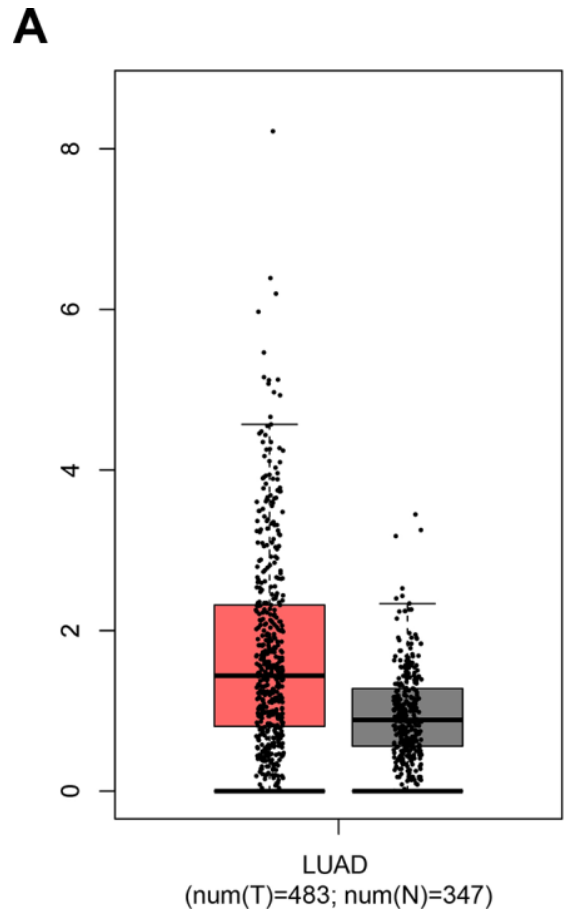

B

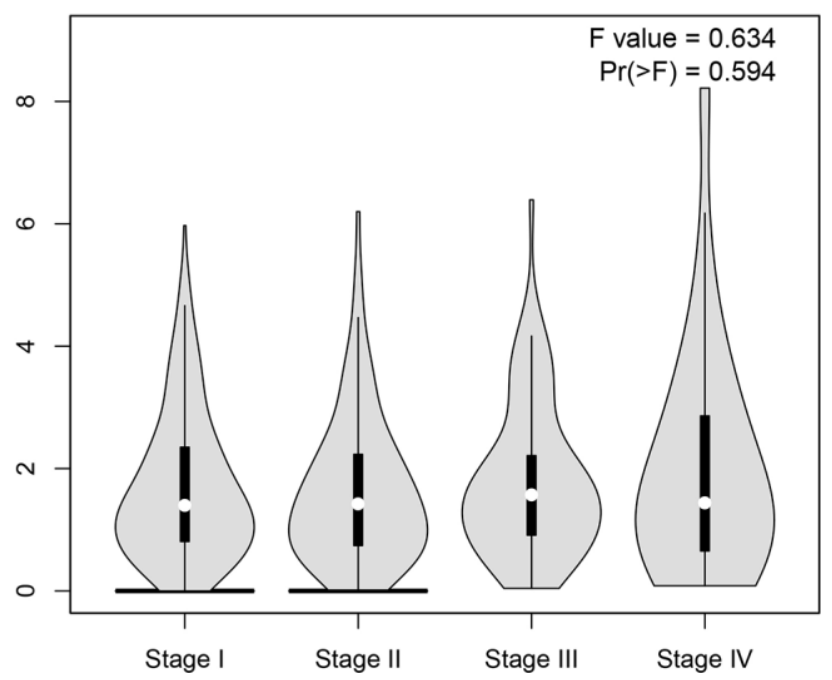

Figure 1. The expression level of ACE2. (A) The box-plot showed the comparison of ACE2 mRNA level in normal tissues vs. LUAD tissues. The red samples represent tissues from LUAD patients and the gray ones represent tissues from healthy persons. (B) The violin plot showed the expression level of ACE2 in different stages of LUAD. The parameters were listed in the upper right. 
The regulatory network revolves around ACE2

The fluctuation of gene expression often involves complicated regulatory networks. To study the potential mechanism of ACE2 dysregulation in LUAD, the relevant molecular around ACE2 were identified. After screening and comparison, a total of 7 differentially expressed correlative genes (DECGs) and 5 differentially expressed correlative miRNAs (DECMs) were detected respectively. In addition, the DECGs (CXCL9, MMP12, IL6, AZU1, FCN3, HYAL1, IRAK3) were involved in multi defense virus processes and the DECMs (miR125b-5p, miR-9-5p, miR-130b-5p, miR-381-3p, miR421) were predicted to target the 3 ' untranslated regions (UTR) of ACE2 (Figure 2 and Supplementary Figure 1).
Among them, CXCL9, MMP12, miR-9-5p, miR-130b5p, miR-381-3p and miR-421 were increased while IL6, AZU1, FCN3, HYAL1, IRAK3 and miR-125b-5p were reduced in LUAD patients. Meanwhile, AZU1, FCN3, HYAL1, IRAK3 and miR-125b-5p were positively correlated with ACE2 while CXCL9, MMP12, IL6, miR-9-5p, miR-130b-5p, miR-381-3p, and miR-421 were negatively correlated (Figure 3). Notably, only miR-125b-5p has an opposite expression trend with ACE2. Thus, miR-125b-5p was assumed to be the single upstream predicted inhibitor of ACE2. In addition, among the 7 DECGs, IL6 owns the highest mutation frequency $(5 \%)$ in LUAD patients might indicate that IL6 could mediate carcinogenesis (Supplementary Figure 2).
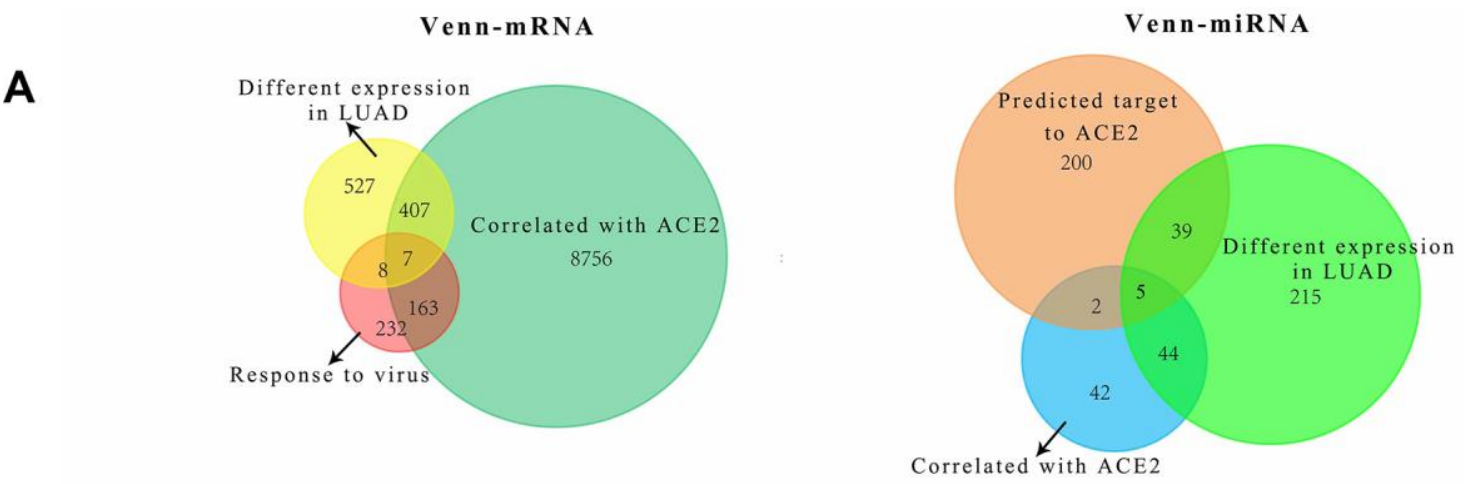

B

CXCL9

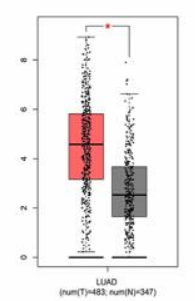

MMP12

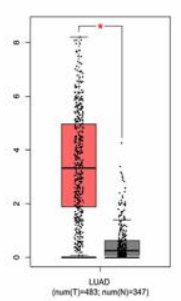

IL 6

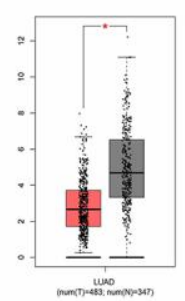

AZU1

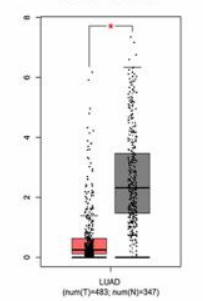

FCN3

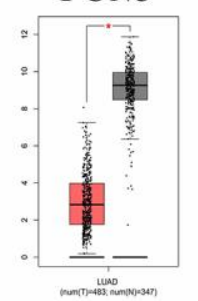

HYAL1

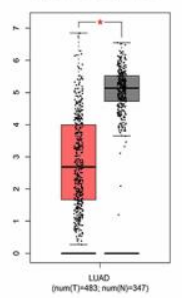

IRAK3

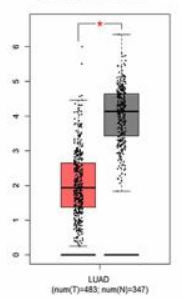

C
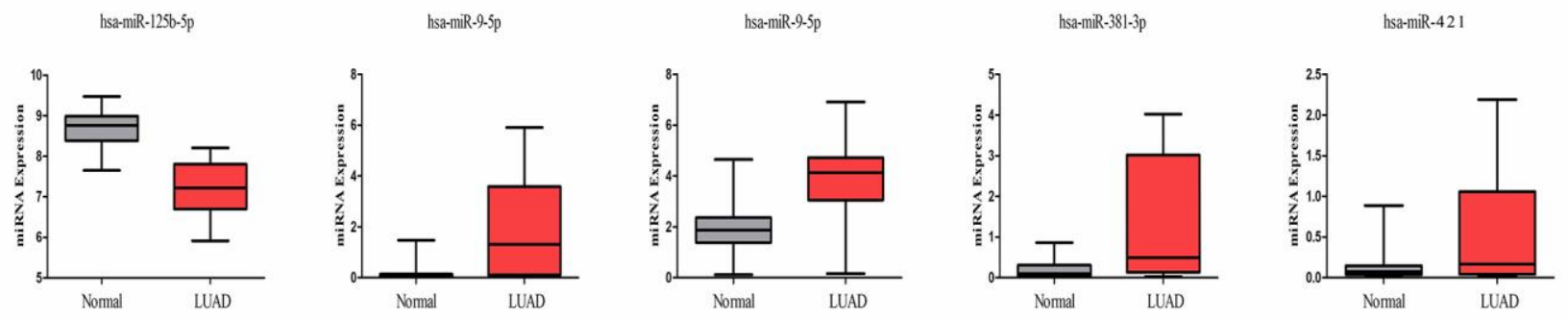

Figure 2. The definition of DECGs and DECMs. (A) Two Venn-plots showed the sifting process of DECGs (left) and DECMs (right). The yellow circle represents the differentially expressed mRNA in LUAD. The green circle represents the genes correlated with ACE 2 in LUAD. The carnation circle represents the gene set involved in virus defense. The orange circle represents the miRNAs which could bind to 3'-UTR of ACE2. The aqua circle represents the differentially expressed miRNAs in LUAD. The blue circle represents the miRNAs correlated with ACE2 in LUAD. (B, C) The box-plots showed the comparison of DECGs or DECMs level in normal tissues vs. LUAD tissues. The red samples represent tissues from LUAD patients and the gray ones represent tissues from healthy persons. 
To understand the involved pathways and functions of the DECGs and DECMs in LUAD, enrichment analysis was performed (Supplementary Figure 3). A total of 41 pathways and $129 \mathrm{GO}$ functions were enriched based on DECGs and DECMs. Interestingly, both 2 DECGs (CXCL9, IL6) and 4 DECMs (miR-125b-5p, miR-9-5p, miR-130b-5p, and miR-421) were enriched in the toll-like receptor signaling pathway (Supplementary Figure 4). In this pathway, CXCL9 takes part in chemotactic effects of T cells while IL6 participates in proinflammatory effects. Then according to the relationships between DECGs, DECMs and virusassociated processes, an interaction network was constructed (Figure 4). Notably, not only ACE2 but IL6 were predicted as the targets of miR-125b-5p. Similarly, both ACE2 and CXCL9 were regarded as the predicted target of miR-9-5p. However, due to the expression trend of CXCL9 and miR-9-5p were not opposite with
ACE2, it was suggested that both CXCL9 and miR-9-5p may not play a decisive role in ACE2 expression.

\section{Immunological function of ACE2 and IL6}

In conclusion, the reduced miR-125b-5p might be the primary inhibitor of ACE2 in LUAD. Once ACE2 was dysregulated, IL6 in toll-like receptor pathway might activate the immune system as a downstream effector. No matter the defense of SARS-CoV-2 or pneumonia in LUAD, the altered immunoreaction was the primary cause. Thus, the ability of ACE2 and IL6 to regulate immune system was evaluated based on the correlation between gene characters (mRNA expression, copy number and methylation) and immune elements (lymphocyte, immune-inhibitor, immune-stimulator, MHC molecule, chemokine, and chemokine receptor) (Figure 5). Interestingly, CXCL9 could mediate
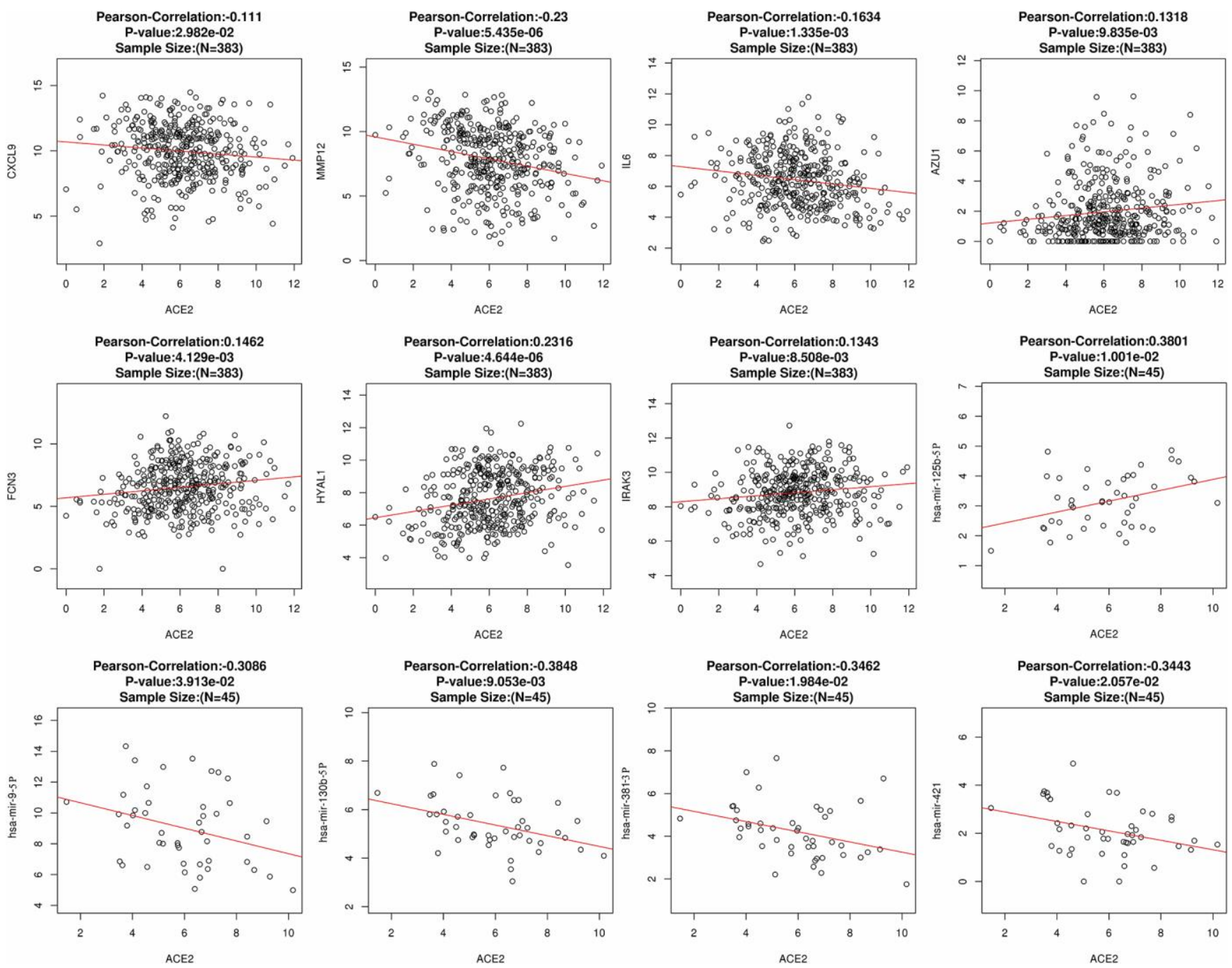

Figure 3. The correlation between ACE2 and DECGs or DECMs. Each hollow circle represents a single sample. The red line represents the correlation. A total of 383 and 45 samples were obtained to calculate the correlation between ACE2 and DECGs or DECMs, respectively. 


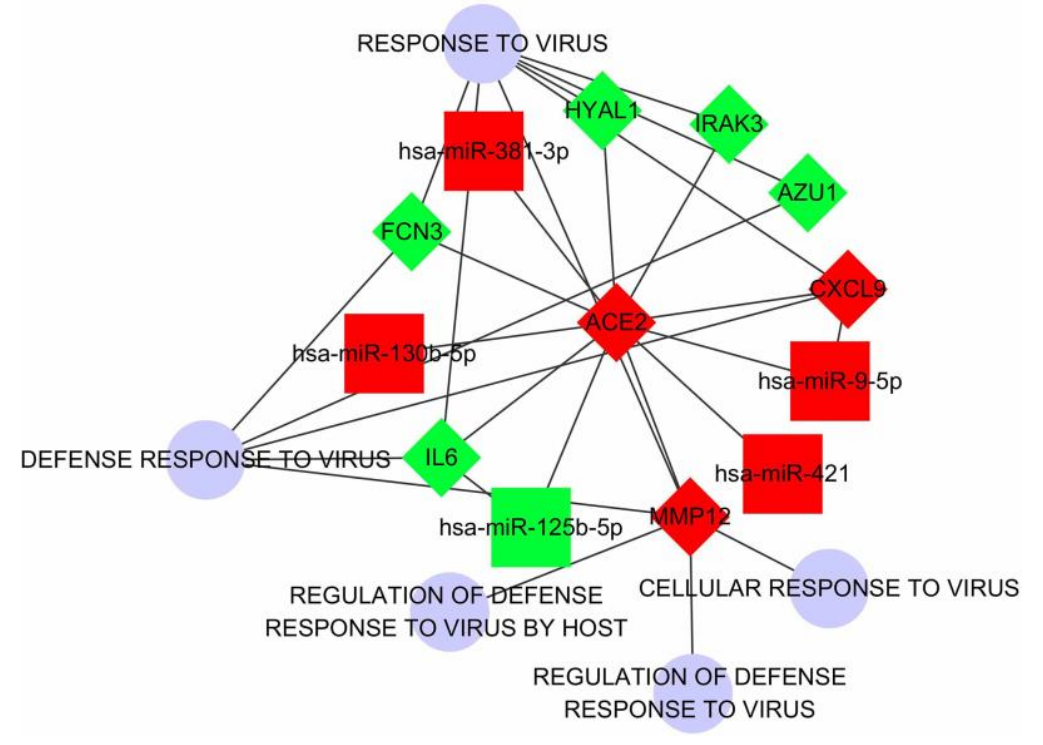

Figure 4. The regulatory network which regards ACE2 as the center. The squares represent DECMs. The rhombus represents DECGs. The blue circles represent biological processes correlated with virus defense. The red nodes represent the up-regulation of DECGs or DECMs. The green nodes represent down-regulation of DECGs or DECMs. The edges between every 2 nodes represent subordination or interactive relationship.

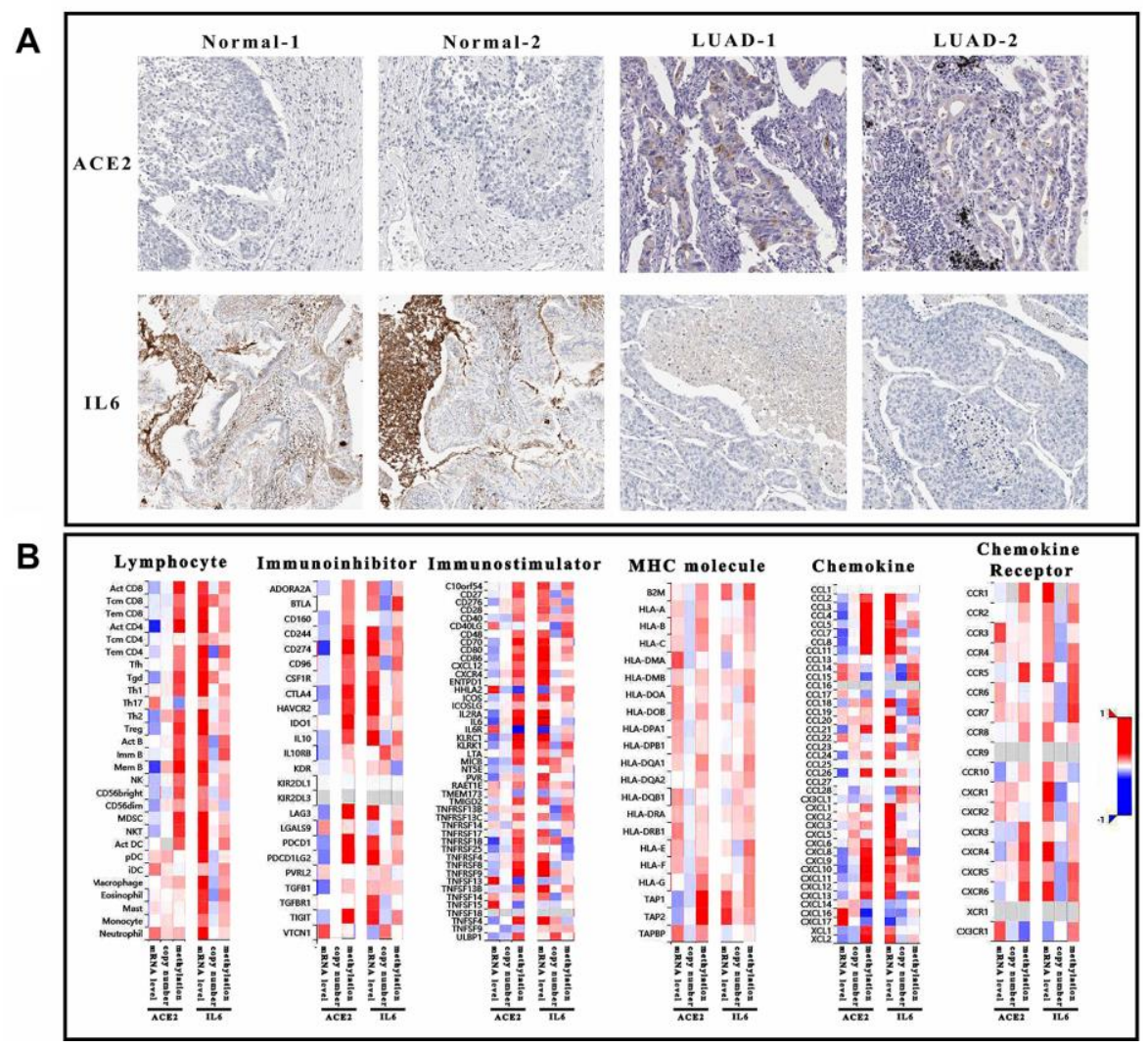

Figure 5. Immunological analysis of ACE2 and IL6. (A) The pathological sections of tumor tissue and normal tissue which was stained by ACE2 and IL6 antibody. Each group has 2 duplicate samples. (B) The heatmap showed the correlation of 6 immunological factors (lymphocyte, immune-inhibitor, immune-stimulator, MHC molecule, chemokine, and chemokine receptor) vs. mRNA expression, copy number and methylation of ACE2 and IL6. 
immunoreaction as a chemokine which also influenced by ACE2 and IL6 (expression level and methylation). Therefore, it was suggested that even CXCL9 might not participate in regulating ACE2 level, it would like to mediate immune-regulation of ACE2 and IL6.

\section{DISCUSSION}

Once infected with SARS-CoV-2, the pathogenic T cells were activated rapidly followed by immune response and virus eliminating. However, the fatal cause of viral pneumonia was the uncontrolled inflammation. In addition, previous study has demonstrated that COVID19 might be more likely to cause an endothelial damage in the vessel system. ACE2, not only as the functional receptor of coronavirus, also emerged as a potent negative regulator of the RAS which maintaining homeostasis of blood pressure and inflammatory responses [27]. Altered activation of the RAS is often attributed to the pathogenesis of many diseases such as hypertension, myocardial infarction and inflammatory lung disease [28, 29]. Compared with normal COVID-19 patients, whether the vessel system of LUAD has higher ACE2 level is unclear. In murine acute respiratory distress syndrome models, it was confirmed that the lack of ACE2 expression in the lung resulted in attenuated vascular permeability, enhanced lung edema, neutrophil infiltration, and further deteriorated lung function [30]. Thus, though higher ACE2 level would increase the risk of SARS-CoV-2 infection, the normal level of ACE2 would also improve prognosis of patients with acute lung injury.

The RAS contained ACE2 participate in maintaining homeostasis of inflammatory responses. However, once SARS-CoV-2 binds to ACE2, the abundance on the cell surface, mRNA expression and the enzymatic activity of ACE2 are significantly reduced which lead to the inflammatory storm. Coincidentally, IL6 was the main factor which could also induce an inflammatory storm. Once infected with SARS-CoV-2, the activated pathogenic $\mathrm{T}$ cells could produce granulocytemacrophage colony-stimulating factors (GM-CSF) and IL6. The GM-CSF could further activate CD14+CD16+ inflammatory monocytes. In addition, more cytokines include IL6 were produced via positive feedback loop. A large concentration of immune cells and tissue fluid in the lungs can block gas exchange between alveoli and capillaries, leading to acute respiratory distress syndrome. Once a cytokine storm forms, the immune system would kill lots of normal cells in the lung while eliminating the virus, severely damaging the lung's ventilation function. In a word, similar to ACE2, IL6 play key role in defense of SARS-CoV-2 with normal expression level. Once deregulated, IL6 will become a lethal factor for patients.
Conventionally, the LUAD patients have lower immunity than healthy persons. The increased ACE2 level in the lung might lead to susceptibility to infection. Nevertheless, the pathology of COVID-19 is complicated, except miRNA introduced in present study, transcription factor, IncRNA and ceRNA may participate in mechanism of COVID. The result of our study may provide a reference for further research.

\section{MATERIALS AND METHODS}

\section{Sequencing datasets}

Dataset GSE74190 was acquired from GEO Dataset. All experiments were approved by the local ethics committee. Expression profiles of 723 human miRNAs were investigated in selected cancerous cells and normal cells populations derived from 36 LUAD and 44 adjacent normal tissues. ALL cells derived from 82 snap-frozen surgical specimens. The sequencing platform is Agilent-019118 Human miRNA Microarray V1 G4470A [miRBase release 9.1 miRNA ID version].

\section{MiRNA target gene prediction and analysis of affected signaling pathway}

The potential target genes of differentially expressed miRNAs in LUAD were acquired from the widely used online databases TargetScanHuman 7.2 [31] and DIANA-microT [32]. To reduce false positives, the predicted target genes which appeared at both databases were accepted. Following this, the list of predicted target genes of individual miRNAs was imported to DIANAmirPath, a miRNA pathway analysis web server. Canonical pathways significantly affected by individual differentially expressed miRNAs were made a contrast and then we obtained the common pathways of which elements were also as the targets of deregulation miRNAs. All these canonical pathways were identified from the Kyoto Encyclopaedia of Genes and Genomes (KEGG) databases.

\section{Microarray data and enrichment analysis}

Total RNA from tissues was isolated using Trizol extractions (Invitrogen). The RNA quantity was assessed by NanoDrop®ND-1000 spectrophotometer and RNA 6000 NanoChips with the Agilent 2100 Bioanalyzer (Agilent, Palo Alto, CA, USA). 100 ng of total RNA was amplified using the Ambion ${ }^{\circledR}$ WT Expression Kit (4411973, Life Technologies). Small RNA libraries were prepared using $1 \mu \mathrm{g}$ of total RNA according to the TruSeq Small RNA Sample Preparation Guide (Illumina, San Diego, CA, USA). To generate count data, the raw sequences were compared to human mature miRNA 
Table1. List of databases.

\begin{tabular}{|c|c|}
\hline Database ID & URL \\
\hline GEO Dataset & https://www.ncbi.nlm.nih.gov/gds/?term= \\
\hline TCGA & https://www.cancer.gov/ \\
\hline cBioportal of cancer genomics & https://www.cbioportal.org/ \\
\hline DSA & http://cancer.digitalslidearchive.net/ \\
\hline The Human Protein Atlas & https://www.proteinatlas.org/ \\
\hline Linked Omics & http://www.linkedomics.org/ \\
\hline Targetscan & http://www.targetscan.org/vert_72/ \\
\hline OncomiR & http://www.oncomir.org/oncomir/index.html \\
\hline DAVID & https://david.ncifcrf.gov/ \\
\hline DIANA-mirPATH v.3 & http://diana.imis.athena-innovation.gr/DianaTools/index.php \\
\hline DIANA-microT & $\begin{array}{c}\text { http://diana.imis.athena- } \\
\text { innovation.gr/DianaTools/index.php?r=microT_CDS/index }\end{array}$ \\
\hline TIMER & https://cistrome.shinyapps.io/timer/ \\
\hline STRING & https://string-db.org/ \\
\hline GEPIA & http://gepia.cancer-pku.cn/index.html \\
\hline Pathview & https://pathview.uncc.edu/ \\
\hline TISIDB & http://cis.hku.hk/TISIDB/index.php \\
\hline
\end{tabular}

sequences (from miRBase version 17) and non-coding RNA sequences (Rfam version 10) by MEGABLAST.

Background deletion, quantile normalization, and probe assembly were performed. Different expression mRNAs between normal vs. LUAD samples were detected by the $\mathrm{R}$ package DESeq [33]. P-values were adjusted for multiple comparisons via using the BenjaminiHochberg procedure [34]. MiRNA with an adjusted pvalue of $<0.05$ and $\operatorname{logFC} \geq 2.0$ was considered as differentially expressed. Gene and miRNA enrichment analyses were performed with DAVID version 6.7 and DIANA- mirPATH v.3 respectively. The enriched biological GO and pathway terms were identified [35]. The interaction network was drawn by Cytoscape. Some other databases used are listed in Table 1.

\section{Statistical analyses}

Results are presented as mean values \pm standard error of the mean (SEM). Unless mentioned otherwise, the statistical comparison between groups was performed by using t-test, a maximum of three comparisons were performed per panel, and robustness of statistical significance was verified after correction for multiple testing. Probability was considered to be significant at $\mathrm{p}<0.05$.

\section{Data availability statement}

Publicly available datasets were analyzed in this study. All data was available.

\begin{abstract}
Abbreviations
COVID-19: coronavirus pneumonia-19; ACE2: angiotensin-converting enzyme 2; Ang II: angiotensin II; Ang 1-7: angiotensin 1-7; RAS: renin-angiotensin system; LUAD: lung adenocarcinoma; NSCLC: non-small cell lung cancer; KEGG: Kyoto Encyclopaedia of Genes and Genomes; SEM: standard error of the mean; MasR: Mas receptor; DECG: differentially expressed correlative gene; DECM: differentially expressed correlative miRNA; RAS: renin-angiotensin system; GM-CSF: granulocytemacrophage colony-stimulating factors.
\end{abstract}

\section{AUTHOR CONTRIBUTIONS}

All authors contributed equally to this work. LC, YL, $\mathrm{HL}$ and LZ contributed to the conception and design of the work. LC analyzed data and wrote this manuscript. $\mathrm{CD}, \mathrm{JT}$ and LZ contributed to interpretation of data for the work. All authors contributed to final approval of the version to be published. All authors agree to be accountable for all aspects of the work

\section{ACKNOWLEDGMENTS}

This study was supported by the sequencing and clinical datasets from TCGA and GEO databases. Thanks for guidance from Pro. Zhong and Dr. Liu.

\section{CONFLICTS OF INTEREST}

The authors declare that they have no conflicts of interest. 


\section{FUNDING}

The Fundamental Research Funds for the Central Universities (Project No. 2020CDJYGSX, 001 To HW Liu) and Series of Evidence Based Study on Hot Spring Wellness, Chongqing Municipal Commission of Culture and Tourism Development (To JL Zhong).

\section{REFERENCES}

1. Xiao X, Chakraborti S, Dimitrov AS, Gramatikoff K, Dimitrov DS. The SARS-CoV S glycoprotein: expression and functional characterization. Biochem Biophys Res Commun. 2003; 312:1159-64.

https://doi.org/10.1016/i.bbrc.2003.11.054 PMID:14651994

2. Moore MJ, Dorfman T, Li W, Wong SK, Li Y, Kuhn JH, Coderre J, Vasilieva N, Han Z, Greenough TC, Farzan M, Choe $\mathrm{H}$. Retroviruses pseudotyped with the severe acute respiratory syndrome coronavirus spike protein efficiently infect cells expressing angiotensinconverting enzyme 2. J Virol. 2004; 78:10628-35. https://doi.org/10.1128/JVI.78.19.10628-10635.2004 PMID:15367630

3. Gallagher TM, Buchmeier MJ. Coronavirus spike proteins in viral entry and pathogenesis. Virology. 2001; 279:371-74.

https://doi.org/10.1006/viro.2000.0757 PMID:11162792

4. Wong SK, Li W, Moore MJ, Choe H, Farzan M. A 193amino acid fragment of the SARS coronavirus $S$ protein efficiently binds angiotensin-converting enzyme 2. J Biol Chem. 2004; 279:3197-201. https://doi.org/10.1074/jbc.C300520200 PMID:14670965

5. Babcock GJ, Esshaki DJ, Thomas WD Jr, Ambrosino DM. Amino acids 270 to 510 of the severe acute respiratory syndrome coronavirus spike protein are required for interaction with receptor. J Virol. 2004; 78:4552-60. https://doi.org/10.1128/jvi.78.9.4552-4560.2004 PMID:15078936

6. Jiang $F$, Yang J, Zhang $Y$, Dong $M$, Wang $S$, Zhang $Q$, Liu $\mathrm{FF}$, Zhang K, Zhang C. Angiotensin-converting enzyme 2 and angiotensin 1-7: novel therapeutic targets. Nat Rev Cardiol. 2014; 11:413-26. https://doi.org/10.1038/nrcardio.2014.59 PMID:24776703

7. Hamming I, Timens W, Bulthuis ML, Lely AT, Navis G, van Goor $\mathrm{H}$. Tissue distribution of ACE2 protein, the functional receptor for SARS coronavirus. A first step in understanding SARS pathogenesis. J Pathol. 2004; 203:631-37.

https://doi.org/10.1002/path.1570 PMID:15141377
8. Kuba K, Imai $Y$, Rao $S$, Gao H, Guo F, Guan B, Huan $Y$, Yang $P$, Zhang $Y$, Deng W, Bao L, Zhang B, Liu G, et al. A crucial role of angiotensin converting enzyme 2 (ACE2) in SARS coronavirus-induced lung injury. Nat Med. 2005; 11:875-79.

https://doi.org/10.1038/nm1267 PMID:16007097

9. Tipnis SR, Hooper NM, Hyde R, Karran E, Christie G, Turner AJ. A human homolog of angiotensinconverting enzyme. Cloning and functional expression as a captopril-insensitive carboxypeptidase. J Biol Chem. 2000; 275:33238-43.

https://doi.org/10.1074/ibc.M002615200 PMID:10924499

10. Li W, Zhang C, Sui J, Kuhn JH, Moore MJ, Luo S, Wong SK, Huang IC, Xu K, Vasilieva N, Murakami A, He Y, Marasco WA, et al. Receptor and viral determinants of SARS-coronavirus adaptation to human ACE2. EMBO J. 2005; 24:1634-43.

https://doi.org/10.1038/sj.emboj.7600640 PMID:15791205

11. Jia HP, Look DC, Tan P, Shi L, Hickey M, Gakhar L, Chappell MC, Wohlford-Lenane C, McCray PB Jr. Ectodomain shedding of angiotensin converting enzyme 2 in human airway epithelia. Am J Physiol Lung Cell Mol Physiol. 2009; 297:L84-96.

https://doi.org/10.1152/ajplung.00071.2009 PMID:19411314

12. Liu Z, Huang XR, Chen HY, Penninger JM, Lan HY. Loss of angiotensin-converting enzyme 2 enhances TGF$\beta /$ smad-mediated renal fibrosis and NF-KB-driven renal inflammation in a mouse model of obstructive nephropathy. Lab Invest. 2012; 92:650-61.

https://doi.org/10.1038/labinvest.2012.2 PMID:22330342

13. Meng Y, Yu CH, Li W, Li T, Luo W, Huang S, Wu PS, Cai SX, Li X. Angiotensin-converting enzyme 2/angiotensin(1-7)/Mas axis protects against lung fibrosis by inhibiting the MAPK/NF-KB pathway. Am J Respir Cell Mol Biol. 2014; 50:723-36.

https://doi.org/10.1165/rcmb.2012-04510C PMID:24168260

14. Zou Z, Yan Y, Shu Y, Gao R, Sun Y, Li X, Ju X, Liang Z, Liu $Q$, Zhao $Y$, Guo F, Bai T, Han $Z$, et al. Angiotensinconverting enzyme 2 protects from lethal avian influenza a H5N1 infections. Nat Commun. 2014; 5:3594.

https://doi.org/10.1038/ncomms4594 PMID:24800825

15. Tao L, Qiu Y, Fu X, Lin R, Lei C, Wang J, Lei B. Angiotensin-converting enzyme 2 activator diminazene aceturate prevents lipopolysaccharide-induced inflammation by inhibiting MAPK and NF-KB pathways in human retinal pigment epithelium. J Neuroinflammation. 2016; 13:35. 
https://doi.org/10.1186/s12974-016-0489-7 PMID:26862037

16. Yamaguchi $M$, Hirai S, Sumi T, Tanaka $Y$, Tada M, Nishii Y, Hasegawa T, Uchida H, Yamada G, Watanabe A, Takahashi $H$, Sakuma $Y$. Angiotensin-converting enzyme 2 is a potential therapeutic target for EGFRmutant lung adenocarcinoma. Biochem Biophys Res Commun. 2017; 487:613-18.

https://doi.org/10.1016/i.bbrc.2017.04.102

PMID:28433633

17. Qian YR, Guo Y, Wan HY, Fan L, Feng Y, Ni L, Xiang Y, Li QY. Angiotensin-converting enzyme 2 attenuates the metastasis of non-small cell lung cancer through inhibition of epithelial-mesenchymal transition. Oncol Rep. 2013; 29:2408-14.

https://doi.org/10.3892/or.2013.2370 PMID:23545945

18. Jia $H$. Pulmonary angiotensin-converting enzyme 2 (ACE2) and inflammatory lung disease. Shock. 2016; 46:239-48.

https://doi.org/10.1097/SHK.0000000000000633 PMID:27082314

19. Li Y, Zhou W, Yang L, You R. Physiological and pathological regulation of ACE2, the SARS-CoV-2 receptor. Pharmacol Res. 2020; 157:104833. https://doi.org/10.1016/j.phrs.2020.104833 PMID:32302706

20. Guan WJ, Ni ZY, Hu Y, Liang WH, Ou CQ, He JX, Liu L, Shan H, Lei CL, Hui DS, Du B, Li L, Zeng G, et al, and China Medical Treatment Expert Group for Covid-19. Clinical characteristics of coronavirus disease 2019 in China. N Engl J Med. 2020; 382:1708-20.

https://doi.org/10.1056/NEJMoa2002032 PMID: $\underline{32109013}$

21. Li C, Lu H. Adenosquamous carcinoma of the lung. Onco Targets Ther. 2018; 11:4829-35.

https://doi.org/10.2147/OTT.S164574

PMID:30147334

22. Myers DJ, Wallen JM. Cancer, Lung Adenocarcinoma, in StatPearls. 2020, StatPearls Publishing StatPearls Publishing LLC.: Treasure Island (FL).

23. Raizada MK, Ferreira AJ. ACE2: a new target for cardiovascular disease therapeutics. J Cardiovasc Pharmacol. 2007; 50:112-19.

https://doi.org/10.1097/FJC.0b013e3180986219 PMID:17703127

24. Bernardi S, Zennaro C, Palmisano S, Velkoska E, Sabato N, Toffoli B, Giacomel G, Buri L, Zanconati F, Bellini G, Burrell LM, De Manzini N, Fabris B. Characterization and significance of ACE2 and Mas receptor in human colon adenocarcinoma. J Renin Angiotensin Aldosterone Syst. 2012; 13:202-09. https://doi.org/10.1177/1470320311426023 PMID:22048948

25. Gallagher PE, Tallant EA. Inhibition of human lung cancer cell growth by angiotensin-(1-7). Carcinogenesis. 2004; 25:2045-52. https://doi.org/10.1093/carcin/bgh236 PMID:15284177

26. Menon J, Soto-Pantoja DR, Callahan MF, Cline JM, Ferrario CM, Tallant EA, Gallagher PE. Angiotensin-(17) inhibits growth of human lung adenocarcinoma xenografts in nude mice through a reduction in cyclooxygenase-2. Cancer Res. 2007; 67:2809-15. https://doi.org/10.1158/0008-5472.CAN-06-3614 PMID:17363603

27. Balakumar P, Jagadeesh G. A century old reninangiotensin system still grows with endless possibilities: AT1 receptor signaling cascades in cardiovascular physiopathology. Cell Signal. 2014; 26:2147-60.

https://doi.org/10.1016/i.cellsig.2014.06.011 PMID:25007996

28. del Castillo Rueda A, Guerrero Sanz JE, Escalante Cobo JL, Grau Carmona T, de Portugal Alvarez J. [Serum and pulmonary angiotensin converting enzyme as a marker of acute lung injury in an experimental model of adult respiratory distress syndrome]. An Med Interna. 1999; 16:229-35.

PMID:10389307

29. Gonzalez NC, Allen J, Schmidt EJ, Casillan AJ, Orth T, Wood JG. Role of the renin-angiotensin system in the systemic microvascular inflammation of alveolar hypoxia. Am J Physiol Heart Circ Physiol. 2007; 292:H2285-94.

https://doi.org/10.1152/ajpheart.00981.2006 PMID:17208999

30. Imai Y, Kuba K, Rao S, Huan Y, Guo F, Guan B, Yang P, Sarao R, Wada T, Leong-Poi $H$, Crackower MA, Fukamizu A, Hui CC, et al. Angiotensin-converting enzyme 2 protects from severe acute lung failure. Nature. 2005; 436:112-16.

https://doi.org/10.1038/nature03712

PMID:16001071

31. Lewis BP, Shih IH, Jones-Rhoades MW, Bartel DP, Burge CB. Prediction of mammalian microRNA targets. Cell. 2003; 115:787-98.

https://doi.org/10.1016/s0092-8674(03)01018-3 PMID:14697198

32. Kiriakidou $M$, Nelson PT, Kouranov A, Fitziev $P$, Bouyioukos C, Mourelatos Z, Hatzigeorgiou A. A combined computational-experimental approach predicts human microRNA targets. Genes Dev. 2004; 18:1165-78. 
https://doi.org/10.1101/gad.1184704

PMID:15131085

33. Anders $S$, Huber W. Differential expression analysis for sequence count data. Genome Biol. 2010; 11:R106.

https://doi.org/10.1186/gb-2010-11-10-r106 PMID:20979621

34. Klipper-Aurbach $\mathrm{Y}$, Wasserman $\mathrm{M}$, BraunspiegelWeintrob N, Borstein D, Peleg S, Assa S, Karp M, Benjamini $Y$, Hochberg $Y$, Laron Z. Mathematical formulae for the prediction of the residual beta cell function during the first two years of disease in children and adolescents with insulin-dependent diabetes mellitus. Med Hypotheses. 1995; 45:486-90.

https://doi.org/10.1016/0306-9877(95)90228-7

PMID: 8748093

35. Huang da W, Sherman BT, Lempicki RA. Systematic and integrative analysis of large gene lists using DAVID bioinformatics resources. Nat Protoc. 2009; 4:44-57.

https://doi.org/10.1038/nprot.2008.211

PMID:19131956 


\section{SUPPLEMENTARY MATERIALS}

\section{Supplementary Figures}

A

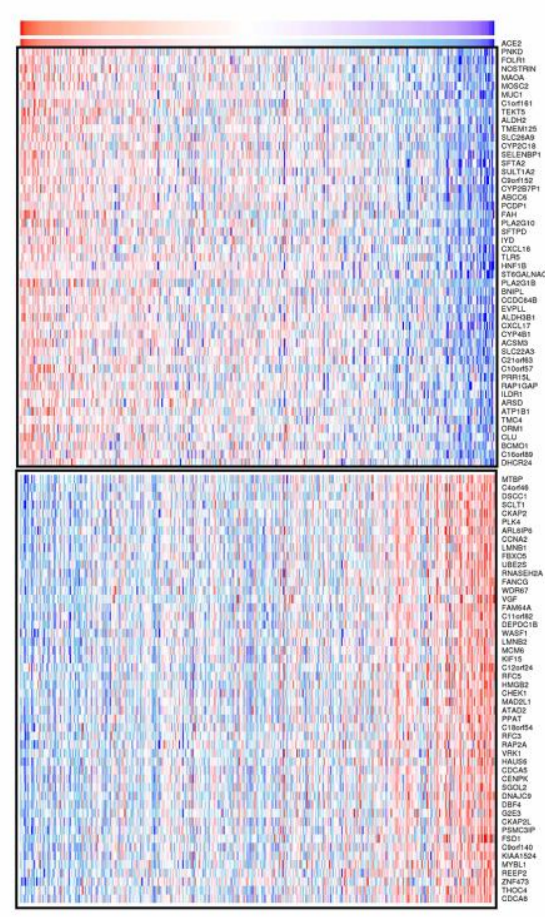

B

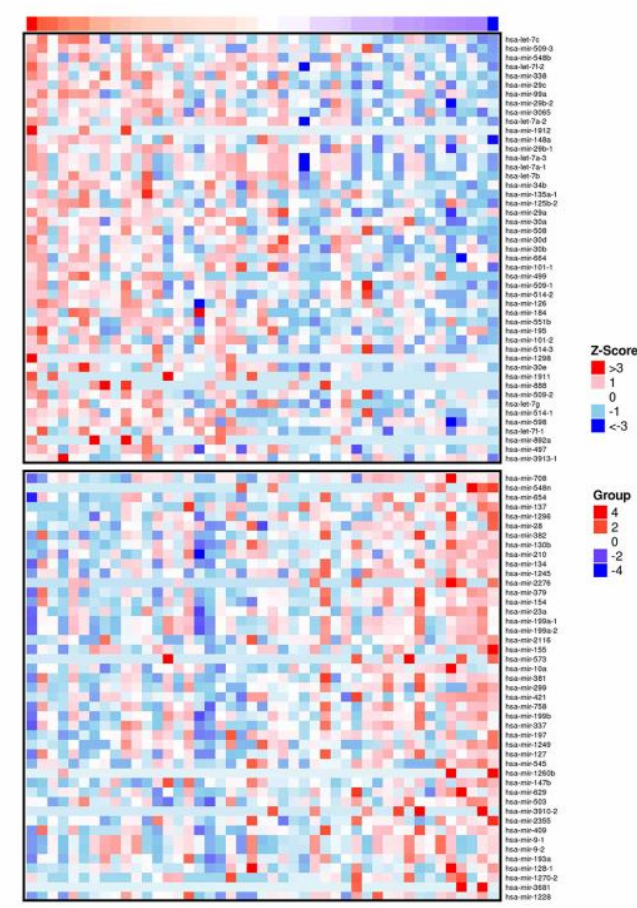

C

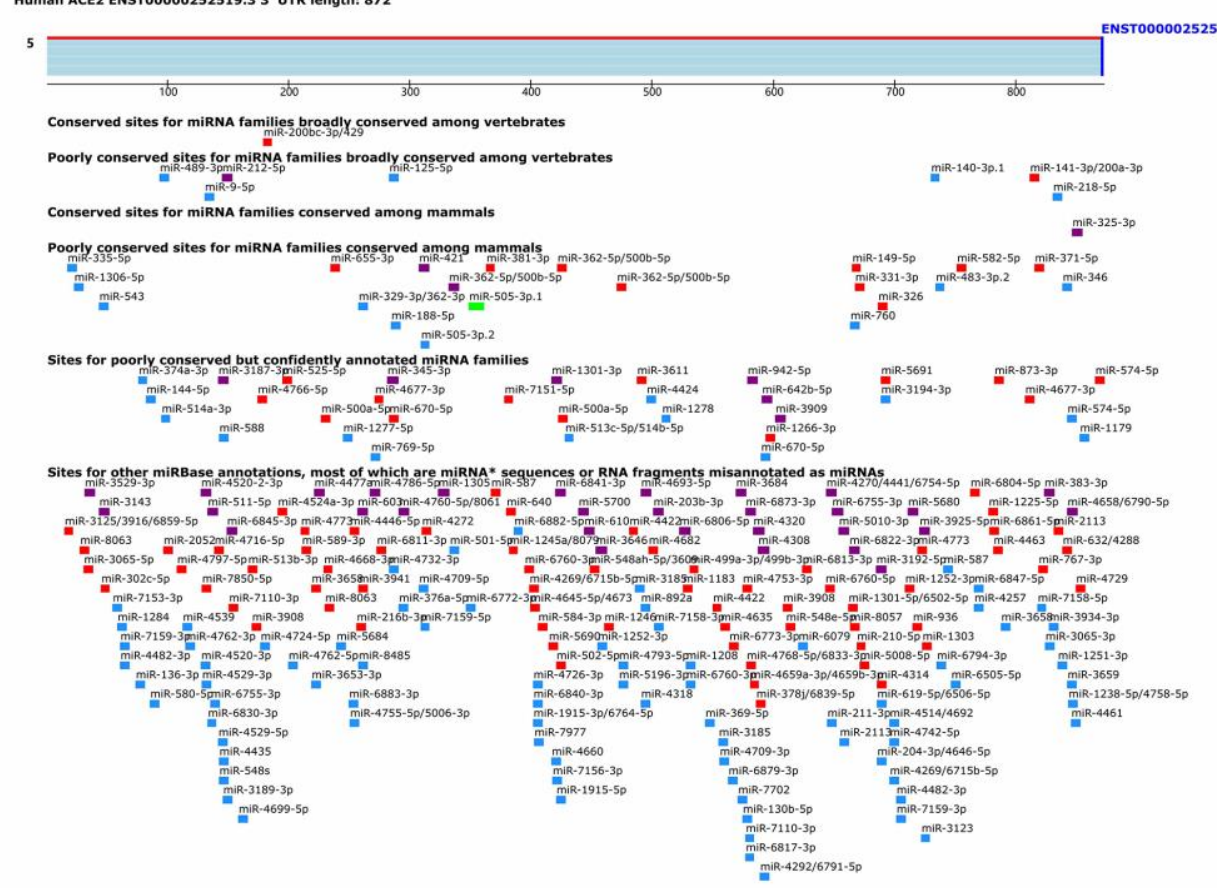

Supplementary Figure 1. The correlated mRNAs (A) and miRNAs (B) of ACE2 in LUAD. Due to the number of correlated genes is too large, only top 50 mRNAs were list. The red point represents positive correlation while the blue ones represents negative correlation. (C) The sketch map of predicted target sites of miRNAs in ACE2 3'-UTR. Different colors represent the site with various probability of preferential conservation. 


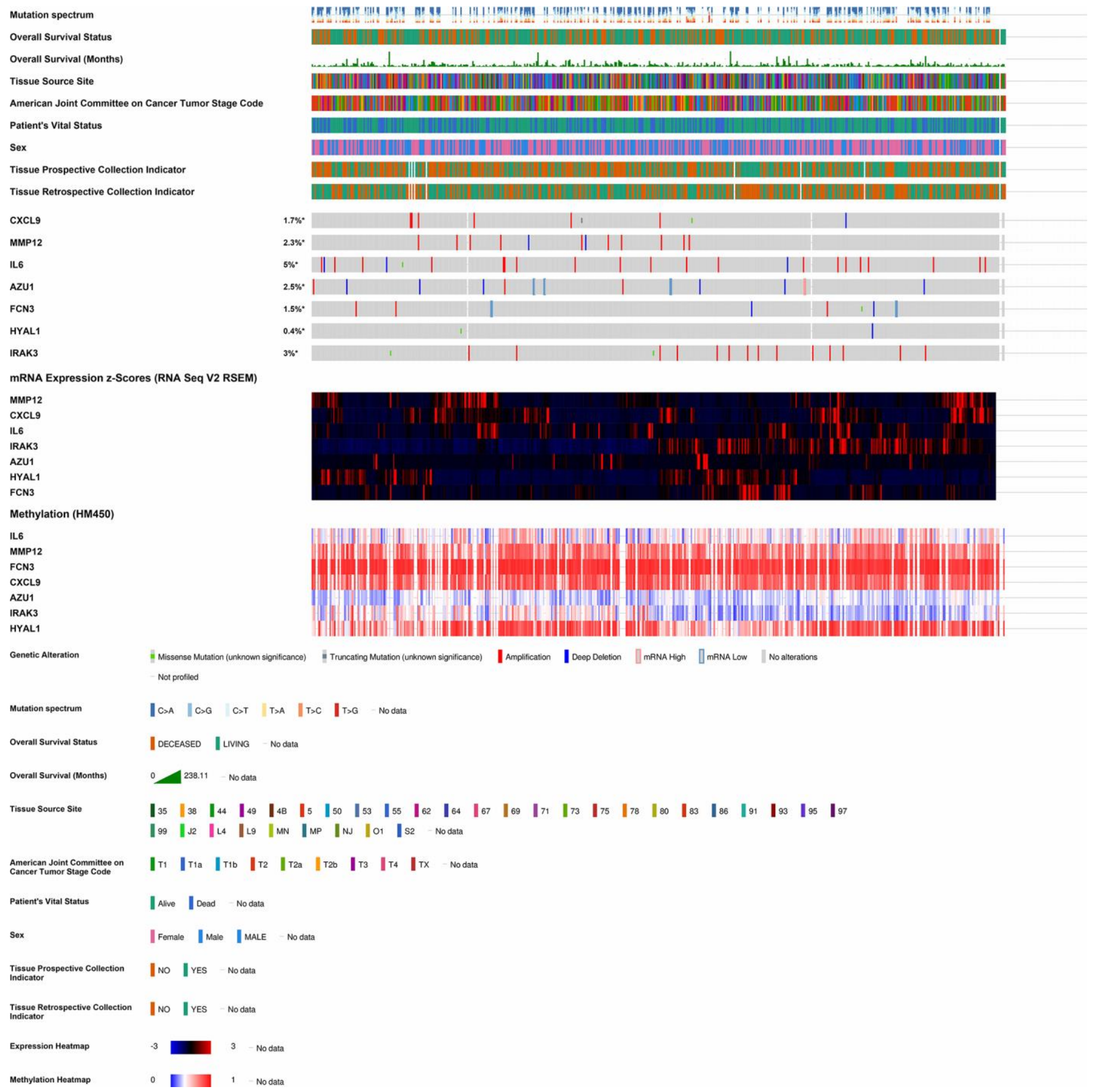

Supplementary Figure 2. Integrated plot of clinical data and 7 mutation of DECGs in 586 LUAD samples. From top to bottom panels indicate: mutation spectrum, overall survival status, overall survival (months), tissue source site, American Joint Committee on Cancer tumor stage code, patients' vital status, sex, tissue prospective collection indicator, tissue retrospective collection indicator, mutation symbol of DECGs, heatmap of DECGs expression and methylation. The key to the color-coding is at the bottom. 
A

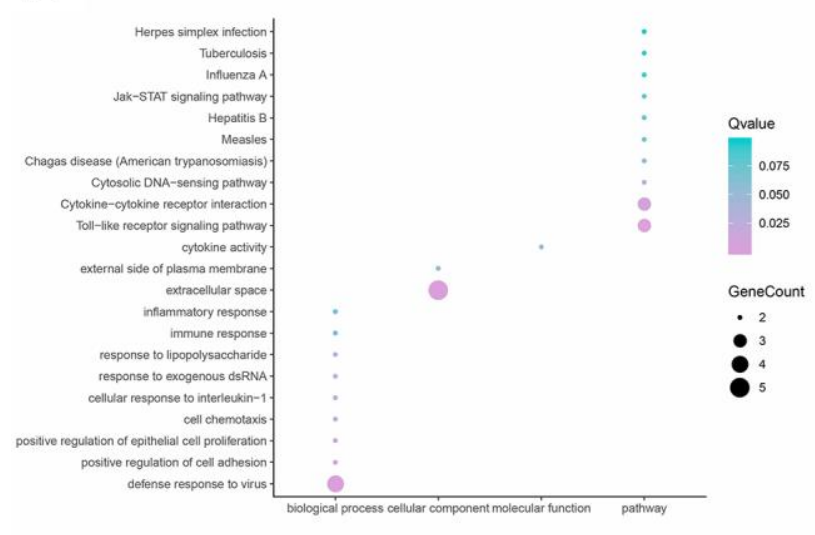

B

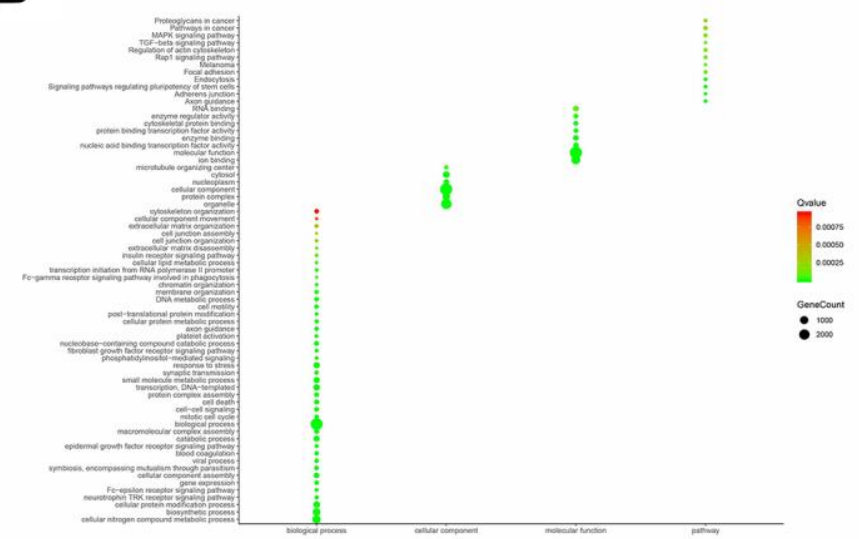

Supplementary Figure 3. The GO enrichment and KEGG pathway analysis of down-regulated (A) and up-regulated (B) genes, respectively. The legends are on the right.



Supplementary Figure 4. The sketch map of toll-like receptor signaling pathway. The red box and green box represent upregulated and down-regulated gene, respectively. 